\title{
Emergency physician use of tissue Doppler bedside echocardiography in detecting diastolic dysfunction: an exploratory study
}

\author{
Marina Del Rios ${ }^{1 *} \mathbb{D}$, Joseph Colla ${ }^{1}$, Pavitra Kotini-Shah ${ }^{1}$, Joan Briller ${ }^{2}$, Ben Gerber ${ }^{3}$ and Heather Prendergast ${ }^{1}$
}

\begin{abstract}
Introduction: This study evaluates the agreement between emergency physician (EP) assessment of diastolic dysfunction (DD) by a simplified approach using average peak mitral excursion velocity $\left(e_{\mathrm{A}}^{\prime}\right)$ and an independent cardiologist's diagnosis of DD by estimating left atrial (LA) pressure using American Society of Echocardiography (ASE) guidelines.

Methods: This was a secondary analysis of 48 limited bedside echocardiograms (LBE) performed as a part of a research study of patients presenting to the Emergency Department (ED) with elevated blood pressure but without decompensated heart failure. EPs diagnosed DD based on $e_{A}^{\prime}<9 \mathrm{~cm} / \mathrm{s}$ alone. A blinded board-certified cardiologist reviewed LBEs to estimate LA filling pressures following ASE guidelines. An unweighted kappa measure was calculated to determine agreement between EP and cardiologist.

Results: Six LBEs were deemed indeterminate by the cardiologist and excluded from the analysis. Agreement was reached in 41 out of 48 cases (85.4\%). The unweighted kappa coefficient was 0.74 (95\% Cl 0.57-0.92). EPs identified 18 out of 20 LBEs diagnosed with diastolic dysfunction by the cardiologist.

Conclusion: There is a good agreement between $\left(e_{A}^{\prime}\right)$ by EP and cardiologist interpretation of LBEs. Future studies should investigate this simplified approach as a one-step method of screening for LV diastolic dysfunction in the ED.
\end{abstract}

\section{Introduction}

Diastolic dysfunction (DD) is an alteration of relaxation, filling, and/or distensibility of the left ventricle [1]. DD can lead to diastolic heart failure and increases the risk of readmission rates and in-hospital mortality [2]. The increased prevalence of DD has led to growing interest in early detection in acute care settings [3, 4].

The American Society of Echocardiography (ASE) guidelines outline a detailed algorithm for the diagnosis of DD which includes (1) spectral pulsed wave Doppler of transmitral inflow; (2) pulsed wave Doppler profile of pulmonary venous flow; (3) mitral annulus downward velocity measurements $(e)$ using tissue Doppler imaging (TDI) at the septum $\left(e_{\mathrm{S}}^{\prime}\right)$ and lateral wall $\left(e_{\mathrm{L}}^{\prime}\right)$; and (4) left atrial

*Correspondence: mdelrios@uic.edu

1 Department of Emergency Medicine, University of Illinois at Chicago, Chicago, Illinois, USA

Full list of author information is available at the end of the article
(LA) volumes [5]. Obtaining these multiple measurements may be time-consuming and difficult for the average EP.

Average peak mitral annulus velocity by TDI $\left(e_{\mathrm{A}}^{\prime}=\left[e_{\mathrm{S}}^{\prime}+e_{\mathrm{L}}^{\prime}\right] / 2\right)$ has been described as an acceptable single-step method for assessing LV relaxation, using $e_{\mathrm{A}}^{\prime}<9 \mathrm{~cm} / \mathrm{s}$ as a threshold [6-9]. TDI measurements can be obtained in 30 s with nearly $100 \%$ success rate, even with poor echocardiographic windows $[10,11]$. This simplified approach may be more suitable for use by EPs with limited experience in echocardiography.

The purpose of this study was to ascertain inter-rater agreement in DD determination between $e_{\mathrm{A}}^{\prime}<9 \mathrm{~cm} / \mathrm{s}$ measured by EPs and cardiologist interpretation of LBEs following the ASE guidelines.

\section{Methods}

\section{Study design}

This was a secondary data set analysis of LBEs completed as part of a prospective, cross-sectional with longitudinal 
follow-up study (details provided elsewhere) of patients presenting to the emergency department (ED) with asymptomatic elevated blood pressure $[12,13]$.

\section{Study protocol and measurements}

LBEs were performed based on research staff availability by EPs (two emergency ultrasound fellowship-trained faculties and one emergency ultrasound fellow) who had performed at least 100 LBEs through routine clinical care and who underwent training and demonstrated proficiency in diastology with a board-certified cardiologist. A sonosite M-Turbo ultrasound system equipped with a harmonic $4.0-\mathrm{MHz}$ variable-frequency phased-array transducer was used to obtain images and measurements. Studies were digitally archived for cardiologist review.

EPs utilized electrocardiogram (EKG) rhythm strips to time diastole. EPs determined $e_{\mathrm{A}}^{\prime}$ by averaging $e_{\mathrm{S}}^{\prime}$ and $e_{\mathrm{L}}^{\prime}$ measurements. EPs considered an $e_{\mathrm{A}}^{\prime}<9 \mathrm{~cm} / \mathrm{s}$ as evidence of DD without adjustment for age or other risk factors. A board-certified cardiologist with an ASE level III echocardiography certification independently reviewed LBE images while blinded to EP interpretation. The cardiologist rated the images in accordance to the 2009 ASE guidelines [8] and upon reviewing digital recordings of the following: parasternal long view for determination of LV wall thickness, apical four-chamber view for estimation of LA size, $E$ and $A$ measurements, $e_{\mathrm{S}}^{\prime}$ and $e_{\mathrm{L}}^{\prime}, E / e^{\prime}$ ratios to assess LA pressure, estimation of LA size, and the EKG rhythm strip (see Table 1 for comparison of data interpretation).

\section{Data analysis}

EPs and cardiologist indicated DD present, DD absent, or indeterminate for each LBE study. A $3 \times 3$ contingency table provided a summary of agreement. Inter-rater reliability between EPs and the cardiologist was determined using an unweighted kappa with 95\% confidence interval (CI) coefficient using Stata Release 15, StataCorp.

\section{Results}

Forty-eight studies were submitted to the cardiologist for review. Cardiologist and EP agreement are summarized in Table 2. Agreement was reached in 41 out of 48 cases (85.4\%). The unweighted kappa coefficient was 0.74 (95\% CI 0.57-0.92).

\section{Discussion}

Diastolic dysfunction is prevalent and delays in diagnosis can lead to increased morbidity and mortality. EPs with focused training in diastology may identify diastolic dysfunction with high sensitivity compared to a cardiologist trained in echocardiography. Previous studies have demonstrated that EPs can identify DD with high sensitivity, but either did not include TDI as part of their assessment [14] or reported only moderate agreement with cardiologist interpretation [4]. One study showed that EPs who met minimum requirements for LBEs based on American College of Emergency Physicians guidelines demonstrated high inter-rater agreement in the assessment of DD using primarily TDI, but failed to compare EP to a cardiologist interpretation [15]. Our study addresses the limitations of previous evidence by demonstrating that by

\begin{tabular}{|c|c|c|c|}
\hline \multirow{2}{*}{$\begin{array}{l}\text { Emergency } \\
\text { physicians }\end{array}$} & \multicolumn{3}{|l|}{ Cardiologist } \\
\hline & DD present & DD not present & Indeterminate $^{a}$ \\
\hline DD present & 18 & 0 & 1 \\
\hline DD not present & 2 & 22 & 4 \\
\hline Indeterminate ${ }^{b}$ & 0 & 0 & 1 \\
\hline
\end{tabular}

a Studies were rated "indeterminate" by cardiology based on the following: fused $E$ and $A$ waves (1), studies that met some criteria but not others (4), and clips with insufficient number of cycles recorded (1)

b One study was rated "indeterminate" by emergency physicians due to extremely disparate $e^{\prime}$ septal and lateral measurements

Table 1 Comparison of data utilized by emergency physician vs. cardiologist for determination of diastolic dysfunction

\begin{tabular}{lll}
\hline Interpretation by & Data collected & Interpretation \\
\hline Emergency physician & TDI measurements mitral annulus (i.e., $e_{S}^{\prime}$ and $e_{L}^{\prime}$ ) & Average TDI velocities at mitral annulus (i.e., $e_{A}^{\prime}$ ) \\
Cardiologist & Sinus EKG rhythm strip & Timing of early and late diastole \\
& Clip of PSL view & LV wall thickness estimation \\
& Clip of apical 4-chamber view & LA diameter \\
& Mitral valve inflow velocities measurements (i.e., $E$ and $A)$ & $E / A$ ratio \\
& TDI measurements mitral annulus (i.e., $e_{S}^{\prime}$ and $\left.e_{L}^{\prime}\right)$ & Ele $e^{\prime}$ to estimate $L A$ pressure \\
& Sinus EKG rhythm strip & E/ $e^{\prime}$ to estimate $L A$ pressure \\
& Timing of early and late diastole
\end{tabular}

TDI, tissue Doppler imaging; PSL, parasternal long, $e_{{ }^{\prime}}^{\prime}$ mitral annulus downward velocity at the septum; $e_{\mathrm{L}}^{\prime}$, mitral annulus downward velocity at the lateral wall; $e_{\mathrm{A}}^{\prime}$, average mitral annulus downward velocity measured $\left(\left[e_{S}^{\prime}+e_{\llcorner}^{\prime}\right] / 2\right) ; E$, peak mitral valve inflow velocity in early diastole; $A$, peak mitral valve inflow velocity in late diastole; LA, left atrium; LV, left ventricle 
following a more simplified approach using $e_{\mathrm{A}}^{\prime}$ alone, EPs can identify DD with high level of agreement compared to a cardiologist following the ASE guidelines.

\section{Limitations}

Our sample size and convenience sampling may have introduced selection bias thus preventing a definitive correlation between $e_{\mathrm{A}}^{\prime}$ and DD. EPs did not screen for regional wall motion abnormalities. Because wall motion abnormalities of the left ventricular basal segments can influence mitral annulus TDI diastolic velocities, this may have led to an overestimation of DD prevalence. Moreover, comparison was limited to cardiologist interpretation of LBE images, which may not be representative of typical exams obtained by a technician or specialist. A larger, multi-center study comparing EP assessment of $e_{\mathrm{A}}^{\prime}$ against performance of a comprehensive echocardiogram can help establish external validity.

\section{Conclusions}

This study highlights a promising simplified approach for identifying DD by EPs. Relying on $e_{\mathrm{A}}^{\prime}$ alone achieved good agreement for determination of DD compared to LBE interpretation by cardiologist. Future studies should further investigate this simplified approach as a onestep method of screening for LV DD in the emergency department.

\section{Authors' contributions}

All authors have contributed to both the collection of data and the writing of the article. All authors read and approved the final manuscript. The authors would like to acknowledge the Research Open Access Publishing (ROAAP) Fund of the University of Illinois at Chicago for financial support towards the open access publishing fee for this article.

\section{Author details}

${ }^{1}$ Department of Emergency Medicine, University of Illinois at Chicago, Chicago, Illinois, USA. ${ }^{2}$ Department of Cardiology, University of Illinois at Chicago, Chicago, Illinois, USA. ${ }^{3}$ Department of Internal Medicine, University of Illinois at Chicago, Chicago, Illinois, USA.

\section{Competing interests}

The authors declare that they have no competing interests.

\section{Availability of data and materials}

This manuscript adhered to institutional policies regarding ultrasonography image collection and research. For access to full dataset, please contact corresponding author by e-mail.

\section{Consent for publication}

All patients gave written consent to participation in the study and the publication of the results.

\section{Ethics approval and consent to participate}

The University of Illinois at Chicago Institutional Review Board approved the study protocol.

\section{Publisher's Note}

Springer Nature remains neutral with regard to jurisdictional claims in published maps and institutional affiliations.

Received: 7 September 2017 Accepted: 9 January 2018

Published online: 25 January 2018

\section{References}

1. Galderisi M (2011) Diagnosis and management of left ventricular diastolic dysfunction in the hypertensive patient. Am J Hypertens 24:507-517

2. Echouffo-Tcheugui JB, Erqou S, Butler J, Yancy CW, Fonarow GC (2016) Assessing the risk of progression from asymptomatic left ventricular dysfunction to overt heart failure: a systematic overview and meta-analysis. JACC Heart Fail 4:237-248

3. Doroudi S, DeLisi MD, DeBari VA (2017) A review of echocardiograms in hypertensive patients greater than 60 years in a community based family medicine program. J Community Hosp Intern Med Perspect 7:28-33

4. Ehrman RR, Russell FM, Ansari AH et al (2015) Can emergency physicians diagnose and correctly classify diastolic dysfunction using bedside echocardiography? Am J Emerg Med 33:1178-1183

5. Nagueh SF, Smiseth OA, Appleton CP et al (2016) Recommendations for the evaluation of left ventricular diastolic function by echocardiography: an update from the American Society of Echocardiography and the European Association of Cardiovascular Imaging. Eur Heart J Cardiovasc Imag 17:1321-1360

6. Nagueh SF, Rao L, Soto J, Middleton KJ, Khoury DS (2004) Haemodynamic insights into the effects of ischaemia and cycle length on tissue Dopplerderived mitral annulus diastolic velocities. Clin Sci 106:147-154

7. Rivas-Gotz C, Manolios M, Thohan V, Nagueh SF (2003) Impact of left ventricular ejection fraction on estimation of left ventricular filling pressures using tissue Doppler and flow propagation velocity. Am J Cardiol 91:780-784

8. Nagueh SF, Appleton CP, Gillebert TC et al (2009) Recommendations for the evaluation of left ventricular diastolic function by echocardiography. Eur J Echocardiogr 10:165-193

9. Lanspa MJ, Gutsche AR, Wilson EL et al (2016) Application of a simplified definition of diastolic function in severe sepsis and septic shock. Crit Care 20:243

10. Farias CA, Rodriguez L, Garcia MJ, Sun JP, Klein AL, Thomas JD (1999) Assessment of diastolic function by tissue Doppler echocardiography: comparison with standard transmitral and pulmonary venous flow. J Am Soc Echocardiogr 12:609-617

11. Kuznetsova T, Thijs L, Knez J, Herbots L, Zhang Z, Staessen JA (2014) Prognostic value of left ventricular diastolic dysfunction in a general population. J Am Heart Assoc 3:e000789

12. Prendergast HM, Colla J, Patel $\mathrm{N}$ et al (2015) Correlation between subclinical heart disease and cardiovascular risk profiles in an Urban emergency Department population with elevated blood pressures: a pilot study. J Emerg Med 48:756-761

13. Prendergast HM, Colla J, Del Rios M, Marcucci J, Schulz R, O'Neal T (2015) Playing a role in secondary prevention in the ED: longitudinal study of patients with asymptomatic elevated blood pressures following a brief education intervention: a pilot study. Public Health 129:604-606

14. Unluer EE, Bayata S, Postaci N et al (2012) Limited bedside echocardiography by emergency physicians for diagnosis of diastolic heart failure. EMJ 29:280-283

15. Saul T, Avitabile NC, Berkowitz R et al (2016) The inter-rater reliability of echocardiographic diastolic function evaluation among emergency physician sonographers. J Emerg Med 51:411-417 